\title{
The random connection model: Connectivity, edge lengths, and degree distributions
}

\section{Srikanth K. Iyer}

Department of Mathematics, Indian Institute of Science, Bangalore,

Karnataka, India

\section{Correspondence}

Srikanth K. Iyer, Department of

Mathematics, Indian Institute of Science,

Bangalore, Karnataka, India.

Email: srikiyer@gmail.com

\section{Funding information}

UGC Center for Advanced Studies (to S.K.I.)

\begin{abstract}
Consider the random graph $G\left(\mathcal{P}_{n}, r\right)$ whose vertex set $\mathcal{P}_{n}$ is a Poisson point process of intensity $n$ on $\left(-\frac{1}{2}, \frac{1}{2}\right]^{d}, d \geq 2$. Any two vertices $X_{i}, X_{j} \in \mathcal{P}_{n}$ are connected by an edge with probability $g\left(\frac{d\left(X_{i}, X_{j}\right)}{r}\right)$, independently of all other edges, and independent of the other points of $\mathcal{P}_{n} . d$ is the toroidal metric, $r>0$ and $g:[0, \infty) \rightarrow[0,1]$ is non-increasing and $\alpha=$ $\int_{\mathbb{R}^{d}} g(|x|) d x<\infty$. Under suitable conditions on $g$, almost surely, the critical parameter $M_{n}$ for which $G\left(\mathcal{P}_{n}, \cdot\right)$ does not have any isolated nodes satisfies $\lim _{n \rightarrow \infty} \frac{\alpha n M_{n}^{d}}{\log n}=1$. Let $\beta=\inf \left\{x>0: x g\left(\frac{\alpha}{x \theta}\right)>1\right\}$, and $\theta$ be the volume of the unit ball in $\mathbb{R}^{d}$. Then for all $\gamma>\beta, G\left(\mathcal{P}_{n},\left(\frac{\gamma \log n}{\alpha n}\right)^{\frac{1}{d}}\right)$ is connected with probability approaching one as $n \rightarrow \infty$. The bound can be seen to be tight for the usual random geometric graph obtained by setting $g=1_{[0,1]}$. We also prove some useful results on the asymptotic behavior of the length of the edges and the degree distribution in the connectivity regime. The results in this paper work for connection functions $g$ that are not necessarily compactly supported but satisfy $g(r)=$ $o\left(r^{-c}\right)$.
\end{abstract}

\section{KEYWORDS}

connectivity, degree distribution, isolated nodes, random connection model, random geometric graphs

\section{1 | INTRODUCTION AND MAIN RESULTS}

The random connection model (RCM) has proved to be a very useful model in applications in many branches of science such as physics, epidemiology, and telecommunications [3]. A specific case of 
the RCM is the random geometric graph (RGG) studied in great detail in [8]. The RGG, the RCM, and its generalizations such as the signal-to-interference-plus-noise ratio (SINR) graph have been used extensively in modeling in wireless networks $[3,4]$. In this context, some of the questions of interest pertain to percolation, connectivity, and coverage. The existence of a phase transition for the percolation problem in a RCM was shown in [7]. Random connection models have also been studied in $[1,2]$. In recent years, there is a growing interest in the study of spatial preferential attachment graphs. Such a graph incorporates elements of the RCM as well as those of preferential attachment graphs. The problem of interest is the asymptotic degree distribution, clustering coefficient, and phase transition behavior (see eg, [5] and references therein).

Mao and Anderson [6] derive a parameter regime under which the number of isolated nodes in the RCM converges to a Poisson distribution when the points are distributed on the unit square and the connection function is integrable. This provides a necessary condition for the RCM to be connected with probability approaching one (also referred to as with high probability (whp)). Penrose [10] classifies connection functions in terms of the rate of decay when the distance is measured on the characteristic length scale of the connection function. Convergence of isolated nodes to a Poisson distribution is shown to hold for any sequence of connection functions that decay exponentially fast uniformly in some power of the distance (as measured on the characteristic length scale) and the expected number of isolated nodes converges to a positive constant. Recently [9] considers a general random connection model and derives conditions under which one obtains Poisson convergence for the number of isolated vertices. In this paper, we derive a strong law result for the critical parameter required to eliminate isolated nodes in a RCM. This critical parameter can be thought of as the analog of the largest nearest neighbor distance in the usual random geometric graph (see [8]).

In case of the RGG, it has been shown that the critical radius required to eleminate isolated nodes is identical to the connectivity threshold $w h p$. This result has been extended for the RCM model in [10] under the condition that the connection functions are zero beyond a certain distance. In this paper, we derive a sufficient condition for the RCM to be connected whp for suitably scaled sequence of connection functions satisfying $g(r)=o\left(r^{-c}\right)$ for some $c>d$. This sufficient condition reduces to the one derived for the RGG. However, for a general RCM, there is a gap in the threshold required to eliminate isolated nodes and the sufficient condition for connectivity derived in this paper. It is an interesting open question whether this gap can be closed for this larger class of connection fuctions.

We also derive some results on the degree distribution in the random connection model in the connectivity regime. Degree distributions have been studied extensively in the theory of random graphs. Given a network, the diameter and the degree distribution are important characteristic that are easy to measure. Often they form an important criterion in evaluating suitability of a model to given data. Unlike the usual RGG where the edges have length smaller than the cutoff radius, in the RCM one can in principle have edges of arbitrary length. The presence of sufficient number of long edges, for instance, can help reduce the number of "hops" required for a packet to reach the destination in a multi-hop wireless network. This fact has been used in what is known as opportunistic routing in wireless networks.

In order to describe some of our results it will be useful to construct a coupled family of graphs. Let $(\Omega, \mathcal{F}, P)$ be a probability space on which all the random variables to be described are defined. Let $S:=\left(-\frac{1}{2}, \frac{1}{2}\right]^{d}$, where $d \geq 2$. We ignore the edge effects in our graphs by equipping $S$ with the toroidal metric $d(x, y)=\inf \left\{|x-y-z|: z \in \mathbb{Z}^{d}\right\}$, where $|\cdot|$ denotes the Euclidean norm. Let $\left\{X_{1}, X_{2}, \ldots\right\}$ be a sequence of independent random variables distributed uniformly in $S$. Let $N_{n}=\sum_{i=1}^{k} Y_{k}$, where $\left\{Y_{k}\right\}_{k \geq 1}$ is a sequence of independent and indentically random variables having the Poisson distribution with mean one. Then $\mathcal{P}_{n}:=\left\{X_{1}, X_{2}, \ldots, X_{N_{n}}\right\}, n \geq 1$ is a sequence of Poisson point processes in $S$ (Proposition 1.5, [8]) with $\mathcal{P}_{n} \subseteq \mathcal{P}_{n+1}$ and $\left(\mathcal{P}_{n+1} \backslash \mathcal{P}_{n}\right)$ independent of $\mathcal{P}_{n}$. The connection function $g:[0, \infty) \rightarrow[0,1]$ is a non-increasing function satisfying $\alpha=\int_{\mathbb{R}^{d}} g(|x|) d x<\infty$. Let $\left\{U_{i j}\right\}_{i, j \geq 1}$ be 
a sequence of independent random variables with uniform distribution on $[0,1]$. The graph $G_{n}(r)=$ $G\left(\mathcal{P}_{n}, r\right)$ is the graph with vertex set $\mathcal{P}_{n}$ with an edge between two vertices $X_{i}, X_{j} \in \mathcal{P}_{n}$ provided $U_{i j} \leq g\left(\frac{d\left(X_{i}, X_{j}\right)}{r}\right)$, independent of every thing else. Thus an edge exists between $X_{i}$ and $X_{j}$ in $G_{n}(r)$ with probability $g\left(\frac{d\left(X_{i}, X_{j}\right)}{r}\right)$ which is non-decreasing in $r$. This together with the coupling constructed above ensures that if $X_{i}, X_{j} \in \mathcal{P}_{n}$ and an edge exists between these two vertices in $G_{n+1}(r)$ then it exists between these two vertices in $G_{n}(s)$ for all $s>r$. With $g=1_{[0,1]}$, the RCM reduces to the usual RGG. Throughout this paper, a coupling will refer to the construction above.

At this point a short discussion on the above construction is in order. To prove strong law results, all the variables need to be defined on the same probability space. The above construction provides a monotonicity that is used only in proving the strong law results. These strong law results yield corresponding results where convergence holds whp. The later results depend only on the distribution of $G_{n}$, and consequently they holds for any arbitrary sequence of graphs $\left\{\tilde{G}_{n}\right\}_{n \geq 1}$ such that $G_{n} \stackrel{d}{=}$ $\tilde{G}_{n}$. The strong law results are somewhat more elegant to state than the convergence $w h p$ results that they imply. Another reason for considering the above coupling is the following. By Lemma 1.4, [8], $P\left(\left|N_{n}-n\right| \geq n^{\frac{3}{4}}\right)$ is summable. Thus, almost surely, the random connection graph with a fixed number $n$ of points is sandwiched between $G_{n-n^{\frac{3}{4}}}$ and $G_{n+n^{\frac{3}{4}}}$ for all $n$ sufficiently large. It follows by the standard de-Poissonization techinique (see eg, [8] Theorem 8.1, [10] Proposition 6.1), strong law results proved for the sequence $\left\{G_{n}\right\}$ can be extended to a sequence of random connection graphs $\left\{\tilde{G}_{n}\right\}$ where $\tilde{G}_{n}$ has $n$ vertices and edges are determined as for $G_{n}$ using the same connection function $g$. In many applications such as in wireless or sensor networks, the number of nodes is varying over time. One can view the snapshot of the network at any point of time as a realization of a RCM model with a fixed number of nodes or from a coupled system of Poisson processes. In such a context the strong law results assure that small fluctuations in the number of nodes will not alter the overall statistical properties of the network in the radius regime in which the strong law result is stated.

For RGG it is known that the critical radius required to connect the graph and that of the largest nearest neighbor distance (the smallest radius required to eliminate isolated nodes) are identical whp. As one increases the radius in a RGG, with high probability, it gets connected at the same time when the isolated nodes disappear (see (13.37) [8]). Thus to study connectivity in a RCM it is instructive to consider the regime under which the expected number of isolated nodes stabilize. Let $W_{n}(r)$ be the number of isolated nodes (vertices with degree zero) in $G_{n}(r)$.

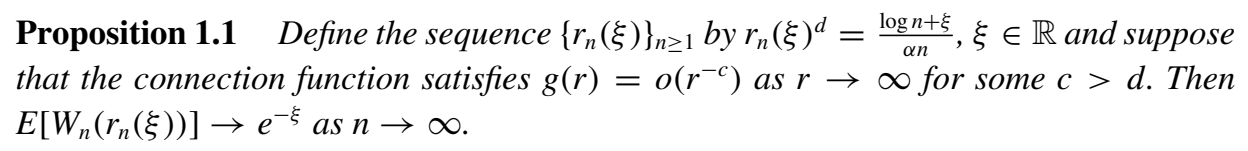

The result also holds if we replace $\xi$ by a sequence $\xi_{n} \rightarrow \xi$. Mao and Anderson [6] in fact show that with $r_{n}(\xi)$ as in Proposition 1.1, $W_{n}$ converges in distribution to a Poisson random variable with mean $e^{-\xi}$. This shows that $\xi_{n} \rightarrow \infty$ is a necessary condition for $G_{n}\left(r_{n}\left(\xi_{n}\right)\right)$ to be connected $w h p$. To state the strong law result for the critical parameter required to eleminate isolated nodes, we define for $n \geq 1$ the sequence of random variables

$$
M_{n}=\inf \left\{r>0: W_{n}(r)=0\right\}
$$

$M_{n}$ is the analog of the largest nearest neighbor distance in a RGG. 
Theorem 1.2 (i) Suppose that $g(r)=o\left(r^{-c}\right)$ as $r \rightarrow \infty$ for some $c>d$. Then almost surely

$$
\limsup _{n \rightarrow \infty} \frac{\alpha n M_{n}^{d}}{\log n} \leq 1
$$

(ii) If $g(r)=o\left(r^{-c}\right)$ as $r \rightarrow \infty$ for some $c>3 d$, then almost surely

$$
\liminf _{n \rightarrow \infty} \frac{\alpha n M_{n}^{d}}{\log n} \geq \frac{c-3 d}{c-d}
$$

The following corollary is an straightforward consequence of Theorem 1.2. It includes for instance, connection functions of the form $g(r)=a e^{-b r}$.

Corollary 1.3 Suppose that $g(r)=o\left(r^{-c}\right)$ as $r \rightarrow \infty$ for every $c>0$. Then almost surely

$$
\lim _{n \rightarrow \infty} \frac{\alpha n M_{n}^{d}}{\log n}=1
$$

Remark 1.4 From Theorem $1.2(i)$ we can conclude that for $r_{n}^{d}=(1+\epsilon) \frac{\log n}{\alpha n}$ and $g(r)=o\left(r^{-c}\right)$ as $r \rightarrow \infty$ for some $c>d, P\left(W_{n}\left(r_{n}\right)=0\right) \rightarrow 0$ as $n \rightarrow \infty$. This assertion does not require that the graphs be coupled. Similarly, from part (ii) of Theorem 1.2 for $r_{n}^{d}=(1-\epsilon) \frac{c-3 d}{c-d} \frac{\log n}{\alpha n}$ and $g(r)=o\left(r^{-c}\right)$ as $r \rightarrow \infty$ for some $c>3 d, P\left(W_{n}\left(r_{n}\right)=0\right) \rightarrow 1$ as $n \rightarrow \infty$. Further, in view of the Poisson convergence result in [6] it would be interesting to know whether the gap in Theorem 1.2 can be closed and whether it continues to hold when $g$ is only given to be integrable.

We are now ready to state a sufficient condition for the graph to be connected. The idea behind the proof is similar to the renormalization arguments used in percolation problems. Let $\theta$ be the volume of the unit ball in $\mathbb{R}^{d}$. Since the function $g$ is non-increasing and takes values in the interval $[0,1]$, the function $x g\left(\frac{\alpha}{x \theta}\right)$ is strictly increasing with limits at zero and infinity being zero and infinity respectively. Thus

$$
\beta=\inf \left\{x>0: x g\left(\frac{\alpha}{x \theta}\right)>1\right\},
$$

is well defined and $\beta \geq 1$. For any $\gamma>0$ and $n \geq 1$, define the sequence $\hat{r}_{n}(\gamma)$ by

$$
\hat{r}(\gamma)^{d}=\frac{\gamma \log n}{\alpha n}
$$

Theorem 1.5 Suppose that the connection function satisfies $g(r)=o\left(r^{-c}\right)$ as $r \rightarrow \infty$ for some $c>d$. Let $\beta$ be as defined in (1.5) and $\hat{r}_{n}(\gamma)$ as in (1.6). Then for any $\gamma>\beta$ the sequence of graphs $G_{n}\left(\hat{r}_{n}(\gamma)\right)$ is connected with probability approaching one as $n \rightarrow \infty$.

Remark 1.6 For the usual random geometric graph obtained by setting $g=1_{[0,1]}$ we see that $\beta=1$. Thus the bound obtained in Theorem 1.5 reduces to the condition required to connect the RGG. However for $g=c 1_{[0,1]}$ where $c \in(0,1)$, we have $\beta=c^{-1}>1$. Thus, for such connection functions, the bound obtained in Theorem 1.5 is not as good as the one obtained in [10]. As noted earlier, the results in [10] hold only when the connection functions have compact support. 


\section{2 | EDGE LENGTHS AND DEGREE DISTRIBUTIONS}

In this section, we prove some results on the length of the longest edge at a typical node and degree distribution. Later in Section 3, Proposition 2.3 we prove a result on the longest edge in the graph in the connectivity regime, that is, when the scaling parameter satisfies (1.6) for some $\gamma>0$. This is a key result that will allow us to localize the computations in the RCM, since apriori, the edges in the $\mathrm{RCM}$ can be arbitrarily long. We also prove strong law results for the minimum and maximum vertex degrees in the connectivity regime.

Our first result in this section is on the length of the longest edge incident on a typical node in $G_{n}\left(\hat{r}_{n}(\gamma)\right)$. Denote the origin by $O$ and let $P^{o}$ denote the Palm distribution of $\mathcal{P}_{n}$ conditioned on a point being located at the origin. Since $\mathcal{P}_{n}$ is a Poisson point process, the distribution of $\mathcal{P}_{n}$ under $P^{o}$ is the same as that of $\mathcal{P}_{n} \cup\{O\}$ under $P$. Let $\hat{r}_{n}(\gamma)$ be as defined in (1.6). We will write $\hat{r}_{n}$ for $\hat{r}_{n}(\gamma)$ whenever there is no ambiguity. Let $B(x, r)$ denote a ball of radius $r$ centered at $x$ with respect to the Euclidean norm denoted by $|\cdot|$. Define

$$
G(a)=\int_{B(O, a)^{c}} g(|z|) d z .
$$

Let $\beta \in \mathbb{R}$. Since $n \hat{r}_{n}^{d} \rightarrow \infty$ as $n \rightarrow \infty$ and $G(a) \rightarrow 0$ as $a \rightarrow \infty$, there exists a sequence $\left\{a_{n}\right\}_{n \geq 1}$ such that

$$
n \hat{r}_{n}^{d} G\left(a_{n}\right) \rightarrow e^{-\beta}
$$

Proposition 2.1 Let $g(r)=o\left(r^{-c}\right)$ as $r \rightarrow \infty$ for some $c>d$. Let $\hat{r}_{n}(\gamma), G(a)$ be as defined in (1.6), (2.1), respectively. Let $\beta \in \mathbb{R}$, and suppose $\left\{a_{n}\right\}_{n \geq 1}$ satisfies (2.2). Define the sequence $\bar{r}_{n}=a_{n} \hat{r}_{n}$. Let $L_{n}^{o}$ be the length of the longest edge incident on the origin in the graph $G_{n}\left(\hat{r}_{n}(\gamma)\right)$ under the Palm measure $P^{o}$. Then

$$
P^{o}\left(L_{n}^{o} \leq \bar{r}_{n}\right) \rightarrow e^{-e^{-\beta}}, \quad \text { as } n \rightarrow \infty
$$

Remark 2.2 The longest edge of a typical node in the RCM is longer by a factor of $a_{n}$ than that in a RGG in the connectivity regime.

To prove part (ii) of Theorem 1.2 we need the following result on the length of the longest edge $L_{n}(\gamma)$ in the graph $G_{n}\left(\hat{r}_{n}\right)$, where $\hat{r}_{n}=\hat{r}_{n}(\gamma)$ is as defined in (1.6). This is a key result that allows us to carry out a localization argument, since a priori the edges in a random connection model with unbounded connection function can be arbitrarily long. Let $D_{n}(r, L)$ be the event that there is an edge in $G_{n}(r)$ of length larger than $L$. For $b>0$ define the sequence

$$
\tilde{L}_{n}(b)=n^{-\frac{b}{d}}, \quad n \geq 1 .
$$

Proposition 2.3 Let $g(r)=o\left(r^{-c}\right)$ as $r \rightarrow \infty$ for some $c>3 d$. Then for any $b \in\left(0, \frac{c-3 d}{c-d}\right)$,

$$
\sum_{n=1}^{\infty} P\left(D_{n}\left(\hat{r}_{n}(\gamma), \tilde{L}_{n}(b)\right)\right)<\infty,
$$

and consequently we have almost surely

$$
\limsup _{n \rightarrow \infty} \frac{\log L_{n}(\gamma)}{\log n} \leq-\frac{c-3 d}{d(c-d)}
$$


We now state some results on the vertex degrees in $G_{n}\left(r_{n}\right)$.

Proposition 2.4 Let $\hat{r}_{n}$ be as in (1.6), $g(r)=o\left(r^{-c}\right)$ as $r \rightarrow \infty$ for some $c>d$. Define $D_{n}(k)$ to be the number of vertices in $G_{n}\left(\hat{r}_{n}\right)$ of degree at least $k$. Then

$$
E\left[\frac{D_{n}\left(k_{n}\right)}{n}\right] \rightarrow 1-\Phi(d)
$$

where $k_{n}=\alpha n \hat{r}_{n}^{d}+d \sqrt{\alpha n \hat{r}_{n}^{d}}$ and $\Phi$ is the standard normal distribution function.

The next two results give strong law asymptotics for the maximum and minimum vertex degrees. Let $H:[0, \infty) \rightarrow[0, \infty)$ be defined by $H(0)=1$, and

$$
H(x)=1-x+x \log x, \quad x>0 .
$$

Note that $H(1)=0, H^{\prime}(x)<0$ for $x<1$ and $H^{\prime}(x)>0$ for $x>1$. Define $H_{+}^{-1}:[0, \infty) \rightarrow[1, \infty)$ to be the inverse of the restriction of $H$ to $[1, \infty)$ and $H_{-}^{-1}:[0,1] \rightarrow[0,1]$ be the inverse of the restriction of $H$ to $[0,1]$.

Theorem 2.5 Let $\Delta_{n}\left(\hat{r}_{n}\right)$ denote the maximum vertex degree in $G_{n}\left(\hat{r}_{n}\right)$ where $\hat{r}_{n}$ is as defined in (1.6).

(i) Suppose that $g(r)=o\left(r^{-c}\right)$ as $r \rightarrow \infty$ for some $c>d$. Then almost surely,

$$
\limsup _{n \rightarrow \infty} \frac{\Delta_{n}\left(\hat{r}_{n}\right)}{\log n} \leq \gamma H_{+}^{-1}\left(\frac{1}{\gamma}\right)
$$

(ii) If $g(r)=o\left(r^{-c}\right)$ as $r \rightarrow \infty$ for some $c>3 d$, then almost surely

$$
\liminf _{n \rightarrow \infty} \frac{\Delta_{n}\left(\hat{r}_{n}\right)}{\log n} \geq \gamma H_{+}^{-1}\left(\frac{c-3 d}{\gamma(c-d)}\right)
$$

The following corollary is an immediate consequence of Theorem 2.5.

Corollary 2.6 Suppose that $g(r)=o\left(r^{-c}\right)$ as $r \rightarrow \infty$ for every $c>0$. Then almost surely,

$$
\lim _{n \rightarrow \infty} \frac{\Delta_{n}\left(\hat{r}_{n}\right)}{\log n}=\gamma H_{+}^{-1}\left(\frac{1}{\gamma}\right)
$$

Thus, in the connectivity regime, the expected vertex degree as well as the maximum degree grows logarithmically in $n$. The following result shows that when the scaling parameter is above the critical threshold required to eleminate isolated nodes, then the minimum vertex degree also grows logarithmically in $n$.

Theorem 2.7 Let $\delta_{n}\left(\hat{r}_{n}\right)$ denote the minimum vertex degree in $G_{n}\left(\hat{r}_{n}\right)$ where $\hat{r}_{n}$ is as defined in (1.6).

(i) If $g(r)=o\left(r^{-c}\right)$ for some $c>3 d$ as $r \rightarrow \infty$, then almost surely $\delta_{n} \rightarrow 0$ as $n \rightarrow \infty$ for any $\gamma<\frac{c-3 d}{c-d}$. 
(ii) If $g(r)=o\left(r^{-c}\right)$ for some $c>d$ as $r \rightarrow \infty$, then for any $\gamma>1$ we have almost surely

$$
\liminf _{n \rightarrow \infty} \frac{\delta_{n}\left(\hat{r}_{n}\right)}{\log n} \geq \gamma H_{-}^{-1}\left(\frac{1}{\gamma}\right)
$$

(iii) If $g(r)=o\left(r^{-c}\right)$ as $r \rightarrow \infty$ for some $c>3 d$, then

$$
\limsup _{n \rightarrow \infty} \frac{\delta_{n}\left(\hat{r}_{n}\right)}{\log n} \leq \gamma H_{-}^{-1}\left(\frac{c-3 d}{\gamma(c-d)}\right)
$$

almost surely for any $\gamma>\frac{c-3 d}{c-d}$.

The following corollary is immediate from Theorem 2.7.

Corollary 2.8 If $g(r)=o\left(r^{-c}\right)$ for every $c>0$ as $r \rightarrow \infty$, then for any $\gamma<1, \delta_{n} \rightarrow 0$ as $n \rightarrow \infty$ almost surely, and for any $\gamma>1$ we have almost surely

$$
\lim _{n \rightarrow \infty} \frac{\delta_{n}\left(\hat{r}_{n}\right)}{\log n}=\gamma H_{-}^{-1}\left(\frac{1}{\gamma}\right)
$$

Remark 2.9 Corollaries 2.6, 2.8 match those obtained for usual random geometric graphs for $g(r)=o\left(r^{-c}\right)$ when $c>3 d$. Question remains open whether the gap in Theorems 2.5, 2.7 can be closed when the connection function satisfies $g(r)=o\left(r^{-c}\right)$ with $d<c \leq 3 d$ as well as the behavior of the maximum and minimum vertex degrees when the function $g$ is only assumed to be integrable.

\section{3 | PROOFS}

In what follows $C_{1}, C_{2}, \ldots$ will denote constants whose values will change from place to place. We recall a few notations. $O$ denotes the origin in $\mathbb{R}^{d}$ and $B(x, r)$ the ball of radius $r$ centered at $x$. $P^{o}$ denotes the Palm distribution of $\mathcal{P}_{n}$, that is the measure $P$ conditioned on there being a point of $\mathcal{P}_{n}$ at the origin and $E^{o}$ the expectation with respect to $P^{o}$. We begin the proofs with a useful Lemma that will be invoked several times.

Lemma 3.1 Let $\left\{r_{n}\right\}_{n \geq 1}$ be a sequence defined by $r_{n}^{d}=\frac{a \log n+b}{\alpha n}$, for some $a>0$ and $b \in \mathbb{R}$. If $g(r)=o\left(r^{-c}\right)$ as $r \rightarrow \infty$ for some $c>d$, then

$$
n r_{n}^{d} \int_{\mathbb{R}^{d} \backslash r_{n}^{-1} S} g(|z|) d z \rightarrow 0, \quad n \rightarrow \infty .
$$

Proof Consider the integral in (3.1).

$$
\begin{aligned}
\int_{\mathbb{R}^{d} \backslash r_{n}^{-1} S} g(|z|) d z & \leq \int_{\mathbb{R}^{d} \backslash B\left(O, r_{n}^{-1} / 2\right)} g(|z|) d z \\
& =C_{1} \int_{r_{n}^{-1} / 2}^{\infty} r^{d-1} g(r) d r \\
& \leq C_{2} \int_{r_{n}^{-1} / 2}^{\infty} r^{d-1-c} d r=C_{3} r_{n}^{c-d}
\end{aligned}
$$

The result now follows since $c>d$. 
Proof of Proposition 1.1 Let $r_{n}=r_{n}(\xi)$ be as defined in the statement of Proposition 1.1. By the Campbell-Mecke formula, $E\left[W_{n}\left(r_{n}\right)\right]$ equals $n$ times the probability that the origin is isolated in $G_{n}\left(r_{n}\right)$ under $P^{o}$. The set of points of $\mathcal{P}_{n}$ with edges incident on $O$ form a Poisson point process with intensity $n g\left(\frac{d(O, \cdot)}{r_{n}}\right)$ over $S$. Consequently

$$
E\left[W_{n}\left(r_{n}\right)\right]=n \exp \left(-n \int_{S} g\left(\frac{d(O, y)}{r_{n}}\right) d y\right) .
$$

Note that $d(O, y)=|y|, y \in S$. Making the change of variable $z=r_{n}^{-1} y$ we get

$$
\begin{aligned}
E\left[W_{n}\left(r_{n}(b)\right)\right] & =n \exp \left(-n r_{n}^{d} \int_{r_{n}^{-1} S} g(|z|) d z\right) \\
& =n \exp \left(-n r_{n}^{d}\left(\alpha-\int_{\mathbb{R}^{d} \backslash r_{n}^{-1} S} g(|z|) d z\right)\right) \\
& =e^{-\xi} \exp \left(n r_{n}^{d} \int_{\mathbb{R}^{d} \backslash r_{n}^{-1} S} g(|z|) d z\right) \rightarrow e^{-\xi} .
\end{aligned}
$$

as $n \rightarrow \infty$ by Lemma 3.1 .

The proof of Theorem 1.2 has two parts which we prove as two separate Propositions.

Proposition 3.2 Let $M_{n}$ be as defined in (1.1). Suppose that $g(r)=o\left(r^{-c}\right)$ as $r \rightarrow \infty$ for some $c>d$. Then almost surely,

$$
\limsup _{n \rightarrow \infty} \frac{\alpha n M_{n}^{d}}{\log n} \leq 1 .
$$

Proof Fix $b>1$ and choose $b_{1} \in(1, b)$ and $a>0$ such that $a\left(b_{1}-1\right)>1$. Let $\hat{r}_{n}$ be as defined in (1.6). For $k \geq 1$ set $n_{k}=k^{a}$ and define the two sequences of events

$$
A_{n}=\left\{W_{n}\left(\hat{r}_{n}(b)\right)>0\right\}, \quad n \geq 1
$$

and

$$
B_{k}=\cup_{n=n_{k}}^{n_{k+1}} A_{n}, \quad k \geq 1 .
$$

Let $\bar{W}_{k}$ be the cardinality of the set

$$
\left\{X \in \mathcal{P}_{n_{k+1}}: \operatorname{deg}(X)=0 \text { in the graph } G\left(\mathcal{P}_{n_{k}} \cup X, \hat{r}_{n_{k+1}}\right)\right\}
$$

If a vertex $X \in \mathcal{P}_{n}$ is isolated in the graph $G_{n}\left(\hat{r}_{n}\right)$ for any $n$ satisfying $n_{k} \leq n \leq n_{k+1}$, then by the coupling it is also isolated in the graph $G\left(\mathcal{P}_{n_{k}} \cup X, \hat{r}_{n_{k+1}}\right)$. Hence

$$
\begin{aligned}
P\left(B_{k}\right) & \leq P\left(\bar{W}_{k}>0\right) \\
& \leq E\left[\sum_{X \in \mathcal{P}_{n_{k+1}}} \mathbf{1}\left\{\operatorname{deg}(X)=0 \text { in the graph } G\left(\mathcal{P}_{n_{k}} \cup X, \hat{r}_{n_{k+1}}\right)\right\}\right] .
\end{aligned}
$$


Applying the Campbell-Mecke formula to the right hand side of the above inequality we get

$$
\begin{aligned}
P\left(B_{k}\right) & \leq n_{k+1} P^{o}\left(O \text { is isolated in } G_{n_{k}}\left(\hat{r}_{n_{k+1}}(b)\right)\right) \\
& =n_{k+1} \exp \left(-n_{k} \int_{S} g\left(\frac{|y|}{\hat{r}_{n_{k+1}}(b)}\right) d y\right) .
\end{aligned}
$$

Make the standard change of variable $z=\hat{r}_{n_{k+1}}(b)^{-1} y$. Since $\frac{n_{k}}{n_{k+1}} \uparrow 1$ as $k \rightarrow \infty$ we have using Lemma 3.1

$$
\begin{aligned}
P\left(B_{k}\right) & \leq C_{1} n_{k+1} \exp \left(-n_{k} \hat{r}_{n_{k+1}}(b)^{d} \alpha\right) \\
& \leq C_{2} n_{k+1} \exp \left(-\frac{n_{k}}{n_{k+1}} b \log n_{k}\right) \\
& \leq \frac{C_{3}}{n_{k}^{b_{1}-1}}=\frac{C_{3}}{k^{a\left(b_{1}-1\right)}},
\end{aligned}
$$

for all $k$ sufficiently large. Hence $P\left(B_{k}\right)$ is summable since $a\left(b_{1}-1\right)>1$. It follows by the Borel-Cantelli lemma that almost surely only finitely many of the events $B_{k}$, and hence the events $A_{n}$, happen. In other words, almost surely

$$
M_{n} \leq \hat{r}_{n}(b) \quad \text { or } \quad \frac{\alpha n M_{n}^{d}}{\log n} \leq b,
$$

eventually for all $b>1$. The result now follows since $b>1$ is arbitrary.

As mentioned earlier, the proof of Theorem 1.2(ii) uses a localization argument that is enabled by Proposition 2.3, which we now proceed to prove.

Proof of Proposition 2.3 Let $A_{n}$ be the set of points in $\mathcal{P}_{n}$ for which there is an edge of length greater than $\tilde{L}_{n}(b)$ incident on it in the graph $G\left(\mathcal{P}_{n}, \hat{r}_{n}(\gamma)\right)$. By the Campbell-Mecke formula and using the fact that $1-e^{-x} \leq x$, we get

$$
\begin{aligned}
P\left(D_{n}\left(\hat{r}_{n}(\gamma), \tilde{L}_{n}(b)\right)\right) & \leq E\left[\sum_{X \in \mathcal{P}_{n}} 1_{A_{n}}(X)\right]=n P^{o}\left(O \in A_{n}\right) \\
& =n\left(1-\exp \left(-n \int_{S \backslash B\left(O, \tilde{L}_{n}(b)\right)} g\left(\frac{|z|}{\hat{r}_{n}(\gamma)}\right) d z\right)\right) \\
& \leq n^{2} \hat{r}_{n}(\gamma)^{d} \int_{\hat{r}_{n}(\gamma)^{-1} S \backslash B\left(O, \hat{r}_{n}(\gamma)^{-1} \tilde{L}_{n}(b)\right)} g(|z|) d z \\
& \leq n^{2} \hat{r}_{n}(\gamma)^{d} \int_{\mathbb{R}^{d} \backslash B\left(O, \hat{r}_{n}(\gamma)^{-1} \tilde{L}_{n}(b)\right)} g(|z|) d z \\
& \leq C_{1} n^{2} \hat{r}_{n}(\gamma)^{d} \int_{\hat{r}_{n}(\gamma)^{-1} \tilde{L}_{n}(b)}^{\infty} r^{d-1} g(r) d r .
\end{aligned}
$$

By the assumption on the function $g$, we have for all $s$ sufficiently large

$$
\int_{s}^{\infty} r^{d-1} g(r) d r \leq C_{2} s^{d-c} .
$$


Since $b<1, \hat{r}_{n}(\gamma)^{-1} \tilde{L}_{n}(b) \rightarrow \infty$ as $n \rightarrow \infty$. Using (3.5) in (3.4) we get for all $n$ sufficiently large

$$
\begin{aligned}
P\left(D_{n}\left(\hat{r}_{n}(\gamma), \tilde{L}_{n}(b)\right)\right) & \leq C_{3} n \log n\left(\hat{r}_{n}(\gamma)^{-1} \tilde{L}_{n}(b)\right)^{d-c} \\
& =C_{4} \frac{(\log n)^{\frac{c}{d}}}{n^{\frac{c}{d}-2-b\left(\frac{c}{d}-1\right)}}
\end{aligned}
$$

which is summable since $b<\frac{c-3 d}{c-d}$. It follows that $P\left(D_{n}\left(\hat{r}_{n}(\gamma), \tilde{L}_{n}(b)\right)\right)$ is summable and by the Borel-Cantelli Lemma almost surely

$$
L_{n}(\gamma) \leq n^{-\frac{b}{d}}
$$

for all $n$ sufficiently large. The result now follows since $b<\frac{c-3 d}{c-d}$ is arbitrary.

Proposition 3.3 Let $M_{n}$ be as defined in (1.1). Suppose that $g(r)=o\left(r^{-c}\right)$ as $r \rightarrow \infty$ for some $c>3 d$, then almost surely

$$
\liminf _{n \rightarrow \infty} \frac{\alpha n M_{n}^{d}}{\log n} \geq \frac{c-3 d}{c-d}
$$

Proof of Proposition 3.3 Fix $\eta \in\left(0, \frac{c-3 d}{c-d}\right)$ and choose $b, b_{1}$ such that $\eta<b_{1}<b<$ $\frac{c-3 d}{c-d}$. Fix $\delta>0$ so that $\delta+\eta<b_{1}$. Define $\tilde{r}_{n}=\tilde{r}_{n}(\delta)$ by $\tilde{r}_{n}^{d}=\frac{\delta \log n}{n \theta}$, where $\theta$ is the volume of the unit ball in $\mathbb{R}^{d}$ and set $\tilde{L}_{n}(b)=n^{-b / d}$.

Roughly speaking, we need to show that the graph $G_{n}(\hat{r}(\eta))$ has isolated nodes. To this end we pack $S$ with balls of radius $\tilde{L}\left(b_{1}\right)$ and consider concentric balls of radius $\tilde{r}_{n}<$ $\tilde{L}_{n}\left(b_{1}\right)$. We will show that at least one of these smaller balls has exactly one point which is isolated. The parameters are so chosen that $\tilde{L}_{n}(b)+\tilde{r}_{n}<\tilde{L}_{n}\left(b_{1}\right)$ and by Proposition 2.3 the length of the edges in the graph will not exceed $\tilde{L}_{n}(b)$. Thus isolation of the vertex falling inside the smaller ball is determined by the points inside the concentric ball of radius $\tilde{L}_{n}\left(b_{1}\right)$ which gives the desired independence to push the computation through. We now make this heuristic argument precise.

Define the sequence of events

$$
D_{n}\left(\hat{r}_{n}(\eta), \tilde{L}_{n}(b)\right)=\left\{L_{n}(\eta)>\tilde{L}_{n}(b)\right\}
$$

where $L_{n}(\eta)$ is the length of the longest edge in the graph $G_{n}\left(\hat{r}_{n}(\eta)\right)$. From Proposition 2.3, we have

$$
\sum_{n=1}^{\infty} P\left(D_{n}\left(\hat{r}_{n}(\eta), \tilde{L}_{n}(b)\right)\right)<\infty .
$$

Let $\kappa_{n}$ be the packing number of $S$ by balls of radius $\tilde{L}_{n}\left(b_{1}\right)$. Then for sufficiently large $n$, we have

$$
\kappa_{n} \geq \frac{C_{1}}{\tilde{L}_{n}\left(b_{1}\right)^{d}}=C_{2} n^{b_{1}}
$$


Let $\left\{x_{1}^{(n)}, x_{2}^{(n)}, \ldots, x_{\kappa_{n}}^{(n)}\right\}$ be a deterministic set of points in $S$ such that the balls $B\left(x_{i}^{(n)}, \tilde{L}_{n}\left(\epsilon_{1}\right)\right), i=1,2, \ldots, \kappa_{n}$ are disjoint. Let $E_{n}^{(i)}\left(\tilde{E}_{n}^{(i)}\right)$ be the event that there is exactly one point of $\mathcal{P}_{n}$ in the ball $B\left(x_{i}^{(n)}, \tilde{r}_{n}\right)$ which is isolated (respectively, has no edge to any point of $\left.\mathcal{P}_{n} \cap B\left(x_{i}^{(n)}, \tilde{L}_{n}\left(b_{1}\right)\right)\right)$ in the graph $G_{n}\left(\hat{r}_{n}(\eta)\right)$.

Suppose we show that

$$
\sum_{n=1}^{\infty} P\left(\cap_{i=1}^{\kappa_{n}}\left(E_{n}^{(i)}\right)^{c}\right)<\infty .
$$

It then follows by the Borel-Cantelli lemma that almost surely, for all $n$ sufficiently large, $E_{n}^{(i)}$ occurs for some $i$. In other words, there is a vertex in $G_{n}\left(\hat{r}_{n}(\eta)\right)$ which is isolated, that is, $M_{n}>\hat{r}_{n}(\eta)$ asymptotically almost surely. The result will then follow since $\eta<\frac{c-3 d}{c-d}$ is arbitrary. Thus it remains to show (3.11).

$$
P\left(\cap_{i=1}^{\kappa_{n}}\left(E_{n}^{(i)}\right)^{c}\right) \leq P\left(\cap_{i=1}^{\kappa_{n}}\left(E_{n}^{(i)}\right)^{c} \cap D_{n}^{c}\right)+P\left(D_{n}\right),
$$

where $D_{n}=D_{n}\left(\hat{r}_{n}(b), \tilde{L}_{n}(b)\right)$ is as defined in (3.8). (3.11) will follow from (3.9) and (3.12) provided we show that

$$
\sum_{n=1}^{\infty} P\left(\cap_{i=1}^{\kappa_{n}}\left(E_{n}^{(i)}\right)^{c} \cap D_{n}^{c}\right)<\infty .
$$

Since $b_{1}<b<1$ we have

$$
\tilde{L}_{n}(b)+\tilde{r}_{n}=n^{-\frac{b}{d}}\left(1+\frac{(\delta \log n)^{\frac{1}{d}}}{\theta^{\frac{1}{d}} n^{\frac{1-b}{d}}}\right) \leq \tilde{L}_{n}\left(b_{1}\right),
$$

for all $n$ sufficiently large. On $D_{n}^{c}$ all edges are of length smaller than $\tilde{L}_{n}(b)$. It follows from (3.13) that

$$
\begin{aligned}
P\left(\cap_{i=1}^{\kappa_{n}}\left(E_{n}^{(i)}\right)^{c} \cap D_{n}^{c}\right) & \leq P\left(\cap_{i=1}^{\kappa_{n}}\left(\tilde{E}_{n}^{(i)}\right)^{c}\right)=\prod_{i=1}^{\kappa_{n}} P\left(\left(\tilde{E}_{n}^{(i)}\right)^{c}\right) \\
& \leq \exp \left(-\kappa_{n} P\left(\tilde{E}_{n}^{(1)}\right)\right)
\end{aligned}
$$

where we have used the fact that the events $\tilde{E}_{n}^{(i)}, i=1,2, \ldots, \kappa_{n}$, are independent and the inequality $1-x \leq e^{-x}$. Set $A_{n}=B\left(x_{n}^{(1)}, \tilde{L}_{n}\left(b_{1}\right)\right) \backslash B\left(x_{n}^{(1)}, \tilde{r}_{n}\right)$. Then using Lemma 3.1, we get

$$
\begin{aligned}
P\left(\tilde{E}_{n}^{(1)}\right) & =n \theta \tilde{r}_{n}^{d} \exp \left(-n \theta \tilde{r}_{n}^{d}\right) \frac{1}{n \theta \tilde{r}_{n}^{d}} \int_{B\left(x_{n}^{(1)}, \tilde{r}_{n}\right)} \exp \left(-n \int_{A_{n}} g\left(\frac{|z-y|}{\hat{r}_{n}(\eta)}\right) d z\right) d y \\
& \geq C_{3} \frac{\log n}{n^{\delta}} \exp \left(-n \hat{r}_{n}(\eta)^{d} \alpha\right) \\
& =C_{3} \frac{\log n}{n^{\delta+\eta}}
\end{aligned}
$$


where the inequality in the second line above is obtained by first making the standard change of variable and then replacing $\hat{r}_{n}^{-1} A_{n}$ by $\mathbb{R}^{d}$. Substituting from (3.10) and (3.15) in (3.14) we get for all $n$ sufficiently large

$$
\begin{aligned}
P\left(\cap_{i=1}^{\kappa_{n}}\left(E_{n}^{(i)}\right)^{c} \cap D_{n}^{c}\right) & \leq \exp \left(-C_{4} n^{b_{1}} \frac{\log n}{n^{\delta+\eta}}\right) \\
& =\exp \left(-C_{4} n^{b_{1}-\delta-\eta} \log n\right),
\end{aligned}
$$

which is summable since $b_{1}>\delta+\eta$. This completes the proof of Proposition 3.3.

Proof of Theorem 1.2 Theorem 1.2 now follows from Propositions 3.2 and 3.3.

Proof of Theorem 1.5 Let $\beta$ be as defined in (1.5). Fix $\gamma>\beta$ and choose $b>1$ such that $\gamma g\left(\frac{b \alpha}{\gamma \theta}\right)>1$. Recall that $\hat{r}_{n}^{d}(\gamma)=\frac{\gamma \log n}{n \alpha}$ and $\tilde{r}_{n}(b)=\frac{b \log n}{n \theta}$. Consider the graph $G_{n}\left(\hat{r}_{n}(\gamma)\right)$. A one hop path is said to exist between $X_{i}, X_{j} \in \mathcal{P}_{n}$ if there is a vertex $X_{k} \in \mathcal{P}_{n}$ such that $\left\{X_{i}, X_{k}\right\}$ and $\left\{X_{k}, X_{j}\right\}$ are edges in $G_{n}\left(\hat{r}_{n}(\gamma)\right)$. Let $E_{n}=E_{n}(\gamma)$ be the event that there is a vertex $X \in \mathcal{P}_{n}$ such that $X$ does not have a one hop path to some point in $\mathcal{P}_{n} \cap B\left(X, \tilde{r}_{n}(b)\right)$ in the graph $G_{n}\left(\hat{r}_{n}(\gamma)\right)$.

Suppose we show that $P\left(E_{n}\right) \rightarrow 0$ as $n \rightarrow \infty$. It follows that every vertex in $G_{n}\left(\hat{r}_{n}(\gamma)\right)$ is connected to each one of its $\tilde{r}_{n}(b)$ neighbor via a one hop path whp. However existence of a path to all the $\tilde{r}_{n}(b)$ neighbor with $b>1$ implies that the graph is connected $w h p$ (Theorem 13.7, [8]). This will prove the result. To this end we estimate $P\left(E_{n}\right)$.

For any $X \in \mathcal{P}_{n}$, let $E_{n}(X)$ be the event that $X$ is not connected to at least one vertex in $\mathcal{P}_{n} \cap B\left(X, \tilde{r}_{n}(b)\right)$ in the graph $G_{n}\left(\hat{r}_{n}(\gamma)\right)$ via a one hop path. Then by the Campbell-Mecke formula we get

$$
P\left(E_{n}\right) \leq E\left[\sum_{X \in \mathcal{P}_{n}} 1_{E_{n}(X)}\right]=n P^{o}\left(E_{n}(O)\right) .
$$

Let $H_{n}$ be the event that the number of points of $\mathcal{P}_{n}$ in $B\left(O, \tilde{r}_{n}(b)\right)$ does not exceed $a \log n$. By the Chernoff bound (Lemma 1.2, [8]), we have

$$
\begin{aligned}
P\left(\mathcal{P}_{n}\left(B\left(O, \tilde{r}_{n}(b)\right)\right) \geq a \log n\right) & \leq \exp \left(-n \theta \tilde{r}_{n}(b)^{d} H\left(\frac{a \log n}{n \theta \tilde{r}_{n}(b)^{d}}\right)\right) \\
& =\frac{1}{n^{b H\left(\frac{a}{b}\right)}} .
\end{aligned}
$$

Since $H(x) \rightarrow \infty$ as $x \rightarrow \infty$, we can choose $a$ large so that $b H\left(\frac{a}{b}\right)>1$. With this choice of $a$ we have

$$
n P\left(H_{n}^{c}\right) \rightarrow 0, \quad \text { as } n \rightarrow \infty .
$$

Let $A_{n}$ be the event that the origin is not connected to a point chosen uniformly at random in the ball $B\left(O, \tilde{r}_{n}(b)\right)$ via a one hop path in the graph $G_{n}\left(\hat{r}_{n}(\gamma)\right)$. Since $P^{o}\left(E_{n}(O)\right) \leq$ $P^{o}\left(E_{n}(O) \cap H_{n}\right)+P^{o}\left(H_{n}^{c}\right)$, by (3.16) and using the union bound, it suffices to show that $n(\log n) P^{o}\left(A_{n}\right) \rightarrow 0$ as $n \rightarrow \infty$.

$$
P^{o}\left(A_{n}\right)=\frac{1}{\theta \tilde{r}_{n}(b)^{d}} \int_{B\left(O, \tilde{r}_{n}(b)\right)} \exp \left(-n \int_{S} g\left(\frac{d(O, y)}{\hat{r}_{n}(\gamma)}\right) g\left(\frac{d(y, z)}{\hat{r}_{n}(\gamma)}\right) d z\right) d y .
$$


The integrand in (3.17) is the probability that there is no point in $\mathcal{P}_{n} \backslash\{O, y\}$ that connects to both the origin and $y$ in $G_{n}\left(\tilde{r}_{n}(\gamma)\right)$ under $P^{o, y}$. Changing the variables from $(y, z)$ to $(u, v)$ with

$$
u=\frac{y}{\hat{r}_{n}(\gamma)} \quad \text { and } \quad v=\frac{z}{\hat{r}_{n}(\gamma)}
$$

in (3.17), and using Lemma 3.1 we get

$$
P^{o}\left(A_{n}\right) \leq C_{2} \frac{\hat{r}_{n}(\gamma)^{d}}{\theta \tilde{r}_{n}(b)^{d}} \int_{B\left(O, \hat{r}_{n}(\gamma)^{-1} \tilde{r}_{n}(b)\right)} \exp \left(-\alpha n \hat{r}_{n}(\gamma)^{d} g(|u|)\right) d u
$$

Since $\hat{r}_{n}(\gamma)^{-1} \tilde{r}_{n}(b)=\frac{b \alpha}{\gamma \theta}$, and $g$ is non-increasing, (3.18) can be bounded by

$$
P^{o}\left(A_{n}\right) \leq C_{2} \exp \left(-\gamma g\left(\frac{b \alpha}{\gamma \theta}\right) \log n\right) .
$$

Consequently

$$
n(\log n) P^{o}\left(A_{n}\right) \leq C_{2} \frac{\log n}{n^{\gamma g\left(\frac{b \alpha}{\gamma \theta}\right)-1}} \rightarrow 0,
$$

since $\gamma g\left(\frac{b \alpha}{\gamma \theta}\right)>1$.

Proof of Proposition 2.1 Recall that $\hat{r}_{n}=\frac{\gamma \log n}{\alpha n}$ and $\bar{r}_{n}=a_{n} \hat{r}_{n}$, where the sequence $\left\{a_{n}\right\}_{n \geq 1}$ satisfies $G\left(a_{n}\right) \rightarrow e^{-\beta}$ as $n \rightarrow \infty$ with $G$ as defined in (2.1). The points of $\mathcal{P}_{n}$ under $P^{o}$ located in $S \backslash B\left(O, \bar{r}_{n}\right)$ that have an edge to the origin form a non-homogenous Poisson point process with intensity $g\left(\frac{|\cdot|}{\hat{r}_{n}}\right)$ and hence

$$
\begin{aligned}
P^{o}\left(L_{n}^{o} \leq \bar{r}_{n}\right) & =\exp \left(-n \int_{S \backslash B\left(O, \bar{r}_{n}\right)} g\left(\frac{|z|}{\hat{r}_{n}}\right) d z\right) \\
& =\exp \left(-n \hat{r}_{n}^{d} \int_{\hat{r}_{n}^{-1} S \backslash B\left(O, \hat{r}_{n}^{-1} \bar{r}_{n}\right)} g(|z|) d z\right) \\
& =\exp \left(-n \hat{r}_{n}^{d} \int_{B\left(O, a_{n}\right)^{c}} g(|z|) d z\right) \exp \left(-n \hat{r}_{n}^{d} \int_{\mathbb{R}^{d} \backslash \hat{r}_{n}^{-1} S} g(|z|) d z\right) \\
& \rightarrow e^{-e^{-\beta}}
\end{aligned}
$$

as $n \rightarrow \infty$ by Lemma 3.1.

Proof of Proposition 2.4 By the Campbell-Mecke formula,

$$
\begin{aligned}
E\left[\frac{D_{n}\left(k_{n}\right)}{n}\right] & =P^{o}\left(\operatorname{deg}(\mathrm{O}) \geq k_{n} \text { in } G_{n}\left(\hat{r}_{n}\right)\right) \\
& =P\left(Z_{n} \geq k_{n}\right),
\end{aligned}
$$

where $Z_{n}$ is a Poisson random variable with mean

$$
m_{n}=n \int_{S} g\left(\frac{|z|}{\hat{r}_{n}}\right) d z=n \hat{r}_{n}^{d} \int_{\hat{r}_{n}^{-1} S} g(|z|) d z
$$

The result now follows by by Lemma 3.1 and the central limit theorem. 
Proof of Theorem 2.5 We first prove (2.8). Fix $\epsilon>0$ and choose $\delta \in(0,1)$ such that $\eta=(1-\delta)(1+\epsilon)-1>0$. Let $n_{k}=k^{a}$ where $a>1$ is chosen such that $a \eta>1$. Define the sequence

$$
c_{n}=\gamma(1+\delta) H_{+}^{-1}\left(\frac{1+\epsilon}{\gamma}\right) \log n .
$$

Suppose the event $\left\{\Delta_{n}\left(\hat{r}_{n}\right) \geq c_{n}\right\}$ occurs for some $n$ satisfying $n_{k} \leq n \leq n_{k+1}$. Then by the coupling the degree of these $N_{n}$ vertices will be higher in the graph $G_{n_{k+1}}\left(\hat{r}_{n_{k}}\right)$. Hence the event $\left\{\Delta_{n_{k+1}}\left(\hat{r}_{n_{k}}\right) \geq c_{n_{k}}\right\}$ will occur. Using this and the Campbell-Mecke formula we get

$$
\begin{aligned}
P\left(\cup_{n=n_{k}}^{n_{k+1}}\left(\Delta_{n}\left(\hat{r}_{n}\right) \geq c_{n}\right)\right) & \leq P\left(\Delta_{n_{k+1}}\left(\hat{r}_{n_{k}}\right) \geq c_{n_{k}}\right) \\
& \leq E\left(\sum_{X \in \mathcal{P}_{n_{k+1}}} \mathbf{1}\left\{\operatorname{deg}(X) \geq c_{n_{k}} \text { in } G_{n_{k+1}}\left(\hat{r}_{n_{k}}\right)\right\}\right) \\
& =n_{k+1} P^{o}\left(\operatorname{deg}(O) \geq c_{n_{k}} \text { in } G_{n_{k+1}}\left(\hat{r}_{n_{k}}\right)\right) .
\end{aligned}
$$

The degree at the origin under $P^{o}$ in $G_{n_{k+1}}\left(\hat{r}_{n_{k}}(\gamma)\right)$ has a Poisson distribution with mean

$$
m_{k}=n_{k+1} \int_{S} g\left(\frac{|z|}{\hat{r}_{n_{k}}(\gamma)}\right) d z
$$

and hence using the Chernoff bound (see Lemma 1.2, [8]) we have

$$
P^{o}\left(\operatorname{deg}(O) \geq c_{n_{k}} \text { in } G_{n_{k+1}}\left(\hat{r}_{n_{k}}\right)\right) \leq \exp \left(-m_{k} H\left(\frac{c_{n_{k}}}{m_{k}}\right)\right) .
$$

By Lemma 3.1 it is easy to see that the sequence $\frac{m_{k}}{n_{k+1} \hat{r}_{n_{k}}^{d}(\gamma)} \rightarrow \alpha$ as $k \rightarrow \infty$. From this and the fact that $\frac{n_{k+1}}{n_{k}} \rightarrow 1$ as $k \rightarrow \infty$ it follows that for all $k$ sufficiently large

$$
(1-\delta) \gamma \log n_{k} \leq m_{k} \leq(1+\delta) \gamma \log n_{k}
$$

From (3.19), (3.23) we get for all $k$ sufficiently large

$$
\frac{c_{n_{k}}}{m_{k}} \geq H_{+}^{-1}\left(\frac{1+\epsilon}{\gamma}\right)
$$

Substituting from (3.23), (3.24) in (3.22) and using the fact that $H$ is increasing in $[1, \infty)$ we get for all $k$ sufficiently large

$$
\begin{aligned}
P^{o}\left(\operatorname{deg}(O) \geq c_{n_{k}} \text { in } G_{n_{k+1}}\left(\hat{r}_{n_{k}}\right)\right) & \leq \exp \left(-(1-\delta) \gamma \log n_{k}\left(\frac{1+\epsilon}{\gamma}\right)\right) \\
& =\exp \left(-(1+\eta) \log n_{k}\right) .
\end{aligned}
$$

Substituting from (3.25) in (3.20) we get for all $k$ sufficiently large

$$
P\left(\cup_{n=n_{k}}^{n_{k+1}}\left(\Delta_{n} \geq c_{n}\right)\right) \leq n_{k+1} \exp \left(-(1+\eta) \log n_{k}\right) \leq C_{1} \frac{1}{k^{a \eta}}
$$


which is summable in $k$. Hence by the Borel-Cantelli lemma, almost surely, only finitely many of the events $\cup_{n=n_{k}}^{n_{k+1}}\left(\Delta_{n} \geq c_{n}\right)$, and hence, only finitely many of the events $\left\{\Delta_{n} \geq c_{n}\right\}$ occur. It follows that almost surely,

$$
\frac{\Delta_{n}}{\log n} \leq \gamma(1+\delta) H_{+}^{-1}\left(\frac{1+\epsilon}{\gamma}\right)
$$

for all $n$ sufficiently large. Since $\epsilon, \delta>0$ are arbitrary and $H_{+}^{-1}$ is increasing, we have that almost surely

$$
\limsup _{n \rightarrow \infty} \frac{\Delta_{n}}{\log n} \leq \gamma H_{+}^{-1}\left(\frac{1}{\gamma}\right)
$$

This proves (2.8).

The proof of (2.9) is similar to that of (3.7) and we will borrow notations used in proving (3.7). Fix $b \in\left(0, \frac{c-3 d}{c-d}\right)$ and choose $b_{1} \in\left(b, \frac{c-3 d}{c-d}\right)$. Fix $\eta>0$ so that $(1+\eta) b<b_{1}$. Let $\tilde{L}_{n}(b)=n^{-\frac{b}{d}}$ and let $D_{n}$ be the event as defined in (3.8). Let $\kappa_{n}$ be the packing number of $S$ by balls of radius $\tilde{L}_{n}\left(b_{1}\right)$ which satisfies the inequality in (3.10) for all $n$ sufficiently large. Let $\left\{x_{1}^{(n)}, x_{2}^{(n)}, \ldots, x_{\kappa_{n}}^{(n)}\right\}$ be a deterministic set of points in $S$ such that the balls $B\left(x_{i}^{(n)}, \tilde{L}_{n}\left(b_{1}\right)\right), i=1,2, \ldots, \kappa_{n}$, are disjoint. Define the sequence $\left\{c_{n}\right\}_{n \geq 1}$ by

$$
c_{n}=\gamma(1-\eta) H_{+}^{-1}\left(\frac{b}{\gamma}\right) \log n .
$$

For $\delta>0$ define $\tilde{r}_{n}=\tilde{r}_{n}(\delta)$ by $\tilde{r}_{n}=\frac{\delta \log n}{n \theta}$, where $\theta$ is the volume of the unit ball in $\mathbb{R}^{d}$ and set $A_{n}=B\left(x_{n}^{(1)}, \tilde{L}_{n}\left(b_{1}\right)\right) \backslash B\left(x_{n}^{(1)}, \tilde{r}_{n}\right)$. For a point of $\mathcal{P}_{n}$ located at $y \in B\left(x_{n}^{(1)}, \tilde{r}_{n}\right)$, the number of edges to points of $\mathcal{P}_{n}$ in $A_{n}$ in the graph $G_{n}\left(\hat{r}_{n}\right)$ has a Poisson distribution with mean

$$
\begin{aligned}
m_{n}(y) & =n \int_{A_{n}} g\left(\frac{|z-y|}{\hat{r}_{n}(\gamma)}\right) d z \\
& =n \hat{r}_{n}(\gamma)^{d} \int_{\hat{r}_{n}(\gamma)^{-1} A_{n}} g(|z-y|) d z .
\end{aligned}
$$

Let

$$
\bar{m}_{n}=\inf _{y \in B\left(x_{n}^{(1)}, \tilde{r}_{n}\right)} m_{n}(y)
$$

It follows from (3.28) that

$$
\lim _{\delta \rightarrow 0} \lim _{n \rightarrow \infty} \frac{\bar{m}_{n}}{n \hat{r}_{n}(\gamma)^{d}}=\lim _{\delta \rightarrow 0} \inf _{y \in B\left(o,\left(\frac{\delta \alpha}{\gamma \theta}\right)^{\frac{1}{d}}\right)} \int_{\mathbb{R}^{d} \backslash B\left(o,\left(\frac{\delta \alpha}{\gamma \theta}\right)^{\frac{1}{d}}\right)} g(|z|) d z=\alpha .
$$

Hence we can and do choose $\delta>0$ sufficiently small so that $b_{1}-(1+\eta) b-\delta>0$ and

$$
\left|\frac{\bar{m}_{n}}{n \hat{r}_{n}(\gamma) \alpha}-1\right| \leq \eta
$$

for all $n$ sufficiently large. 
Let $E_{n}^{(i)}\left(\tilde{E}_{n}^{(i)}\right)$ be the event that there is exactly one point of $\mathcal{P}_{n}$ in the ball $B\left(x_{i}^{(n)}, \tilde{r}_{n}\right.$ ) whose degree is at least $c_{n}$ (which has an edge to at least $c_{n}$ many points in $\left.\mathcal{P}_{n} \cap B\left(x_{i}^{(n)}, \tilde{L}_{n}\left(b_{1}\right)\right)\right)$ in the graph $G_{n}\left(\hat{r}_{n}(\gamma)\right)$. Suppose we show that

$$
\sum_{n=1}^{\infty} P\left(\cap_{i=1}^{\kappa_{n}}\left(E_{n}^{(i)}\right)^{c}\right)<\infty .
$$

It then follows by the Borel-Cantelli lemma that almost surely, for all $n$ sufficiently large, $E_{n}^{(i)}$ occurs for some $i$. In other words, there is a vertex in $G_{n}\left(\hat{r}_{n}(\gamma)\right)$ whose degree is at least $c_{n}$. (2.9) then follows since $b<\frac{c-3 d}{c-d}, \eta>0$ are arbitrary small and $H_{+}^{-1}$ is increasing.

Following the same steps as in the proof of (3.11), it suffices to show that $\exp \left(-\kappa_{n} P\left(\tilde{E}_{n}^{(1)}\right)\right)$ is summable.

Let $\operatorname{Po}(\mu)$ denote a Poisson random variable with mean $\mu$. By definition of $\bar{m}_{n}$, Lemma 1.3, [8] and (3.30) we have for all $n$ sufficiently large

$$
\begin{aligned}
P\left(\tilde{E}_{n}^{(1)}\right) & \geq n \theta \tilde{r}_{n}^{d} \exp \left(-n \theta \tilde{r}_{n}^{d}\right) P\left(P o\left(\bar{m}_{n}\right) \geq c_{n}\right) \\
& \geq \frac{\delta \log n}{n^{\delta}}\left(\frac{e^{-1 /\left(12 c_{n}\right)}}{\sqrt{2 \pi c_{n}}}\right) \exp \left(-\bar{m}_{n} H\left(\frac{c_{n}}{\bar{m}_{n}}\right)\right) \\
& \geq C_{1} \frac{(\log n)^{\frac{1}{2}}}{n^{\delta}} \exp \left(-(1+\eta) \gamma \log n H\left(\frac{c_{n}}{(1-\eta) \gamma \log n}\right)\right) \\
& =C_{1} \frac{(\log n)^{\frac{1}{2}}}{n^{\delta+(1+\eta) b}} .
\end{aligned}
$$

It follows from (3.10) and the above inequality that

$$
\begin{aligned}
\exp \left(-\kappa_{n} P\left(\tilde{E}_{n}^{(1)}\right)\right) & \leq \exp \left(-C_{4} n^{b_{1}} \frac{(\log n)^{\frac{1}{2}}}{n^{(1+\eta) b+\delta}}\right) \\
& =\exp \left(-C_{4} n^{b_{1}-(1+\eta) b-\delta}\right)
\end{aligned}
$$

which is summable since $b_{1}-(1+\eta) b-\delta>0$. This proves (2.10) since $\epsilon_{1}>0$ is arbitrary.

Proof of Theorem 2.7 Part (i) of the proof follows immediately from Theorem 1.2 (ii). The rest of the proof is entirely analogous to the proof of Theorem 2.5 with the obvious changes. We illustrate this by proving part (ii), the proof of which is similar to that of (2.8).

Fix $\epsilon>0$ so that $\frac{1+\epsilon}{\gamma}<1$ and choose $\delta \in(0,1)$ such that $\eta=(1-\delta)(1+\epsilon)-1>0$. Let $n_{k}=k^{a}$ where $a>1$ is chosen such that $a \eta>1$. Define the sequence

$$
c_{n}=(1-\delta) \gamma H_{-}^{-1}\left(\frac{1+\epsilon}{\gamma}\right) \log n
$$

Let $\bar{\delta}_{k}$ be the minimum over all $X \in \mathcal{P}_{n_{k+1}}$ of $\operatorname{deg}(X)$ in the graph $G\left(\mathcal{P}_{n_{k}} \cup\{X\}, \hat{r}_{n_{k+1}}\right)$. By the coupling, for any $n$ satisfying $n_{k} \leq n \leq n_{k+1}$ the $\operatorname{deg}(X)$ for any $X \in \mathcal{P}_{n}$ in the graph $G_{n}\left(\hat{r}_{n}\right)$ is larger than $\operatorname{deg}(X)$ in the graph $G\left(\mathcal{P}_{n_{k}} \cup\{X\}, \hat{r}_{n_{k+1}}\right)$. Thus if the event $\left\{\delta_{n} \leq c_{n}\right\}$ 
occurs then so does the event $\left\{\bar{\delta}_{k} \leq c_{n_{k}}\right\}$. Using this and the Campbell-Mecke formula we get

$$
\begin{aligned}
P\left(\cup_{n=n_{k}}^{n_{k+1}}\left(\delta_{n}\left(\hat{r}_{n}\right) \leq c_{n}\right)\right) & \leq P\left(\bar{\delta}_{k} \leq c_{n_{k}}\right) \\
& \leq E\left(\sum_{X \in \mathcal{P}_{n_{k+1}}} \mathbf{1}\left\{\operatorname{deg}(X) \leq c_{n_{k}} \text { in } G\left(\mathcal{P}_{n_{k}} \cup\{X\}, \hat{r}_{n_{k+1}}\right)\right)\right. \\
& =n_{k+1} P^{o}\left(\operatorname{deg}(O) \leq c_{n_{k}} \text { in } G_{n_{k}}\left(\hat{r}_{n_{k+1}}\right)\right) .
\end{aligned}
$$

The degree at the origin under $P^{o}$ in $G_{n_{k}}\left(\hat{r}_{n_{k+1}}(\gamma)\right)$ is Poisson distributed with mean

$$
m_{k}=n_{k} \int_{S} g\left(\frac{|z|}{\hat{r}_{n_{k+1}}(\gamma)}\right) d z
$$

and hence using the Chernoff bound (see Lemma 1.2, [8]) we have

$$
P^{o}\left(\operatorname{deg}(O) \leq c_{n_{k}} \text { in } G_{n_{k+1}}\left(\hat{r}_{n_{k}}\right)\right) \leq \exp \left(-m_{k} H\left(\frac{c_{n_{k}}}{m_{k}}\right)\right)
$$

As noted above (3.23), $\frac{n_{k+1}}{n_{k}} \rightarrow 1$ and $\frac{m_{k}}{n_{k} r_{n_{k+1}}^{d}(\gamma)} \rightarrow \alpha$ as $k \rightarrow \infty$. It follows that for all $k$ sufficiently large

$$
(1-\delta) \gamma \log n_{k} \leq m_{k} \leq(1+\delta) \gamma \log n_{k}
$$

and hence

$$
\frac{c_{n_{k}}}{m_{k}} \leq H_{-}^{-1}\left(\frac{1+\epsilon}{\gamma}\right) .
$$

Substituting from (3.37), (3.38) in (3.36) and using the fact that $H$ is decreasing in $(0,1]$ we get

$$
\begin{aligned}
P^{o}\left(\operatorname{deg}(O) \leq c_{n_{k}} \text { in } G_{n_{k+1}}\left(\hat{r}_{n_{k}}\right)\right) & \leq \exp \left(-(1-\delta) \gamma\left(\log n_{k}\right)\left(\frac{1+\epsilon}{\gamma}\right)\right) \\
& =\exp \left(-(1+\eta) \log n_{k}\right) .
\end{aligned}
$$

Substituting from (3.39) in (3.34) we get

$$
\begin{aligned}
P\left(\cup_{n=n_{k}}^{n_{k+1}}\left(\delta_{n} \leq c_{n}\right)\right) & \leq n_{k+1} \exp \left(-(1+\eta) \log n_{k}\right) \\
& \leq C_{1} \frac{1}{k^{a \eta}},
\end{aligned}
$$

which is summable in $k$. Hence by the Borel-Cantelli lemma, almost surely, only finitely many of the events $\cup_{n=n_{k}}^{n_{k+1}}\left(\delta_{n} \leq c_{n}\right), k \geq 1$, and hence, only finitely many of the events $\left\{\delta_{n} \leq c_{n}\right\}, n \geq 1$ occur. It follows that almost surely,

$$
\frac{\delta_{n}}{\log n} \geq(1-\delta) \gamma H_{-}^{-1}\left(\frac{1+\epsilon}{\gamma}\right)
$$


for all $n$ sufficiently large. Since $\epsilon, \delta>0$ are arbitrary and $H_{-}^{-1}$ is decreasing, we have that almost surely

$$
\liminf _{n \rightarrow \infty} \frac{\delta_{n}}{\log n} \geq \gamma H_{-}^{-1}\left(\frac{1}{\gamma}\right)
$$

This proves (2.11).

\section{ACKNOWLEDGMENT}

The author would like to thank two anonymous referees for bringing to notice the paper of [10] as well as numerous suggestions that have improved the paper.

\section{REFERENCES}

[1] P. Ballister, B. Bollobás, and M. Walters, Continuum percolation with steps in an annulus, Ann. Appl. Probab. 14 (2004), 1869-1879.

[2] P. Ballister, B. Bollobás, and M. Walters, Continuum percolation with steps in the square or the disk, Random S truct. Algorithms 26 (2005), no. 4, 392-403.

[3] M. Franceschetti and R. Meester, Random networks for communication, Cambridge University Press, Cambridge, 2007.

[4] M. Haenggi, Stochastic geometry for wireless networks, Cambridge University Press, Cambridge, 2013.

[5] E. Jacob and P. Mörters, Spatial preferential attachment networks: Power laws and clustering coefficients, Ann. Appl. Probab. 25 (2015), no. 2, 632-662.

[6] G. Mao and B. D. Anderson, On the asymptotic connectivity of random networks under the random connection model, Proceedings of IEEE INFOCOM, Shangai, China, 2011, pp. 631-639.

[7] M. D. Penrose, On a continuum percolation model, Adv. Appl. Probab. 23 (1991), no. 3, 536-556.

[8] M. D. Penrose, Random geometric graphs, Oxford University Press, Oxford, 2003.

[9] M. D. Penrose, Inhomogenous random graphs, isolated vertices and Poisson approximation, arXiv:1507.07132 [math.PR], 2015.

[10] M. D. Penrose, Connectivity of soft random geometric graphs, Ann. Appl. Probab. 26 (2016), no. 2, 986-1028.

How to cite this article: Srikanth $\mathrm{K}$. Iyer. The random connection model: Connectivity, edge lengths, and degree distributions. Random Struct Alg. 2018;52:283-300. https://doi.org/10.1002/rsa.20741 